\title{
DIRECTIONAL-DISRUPTIVE SELECTION IN DROSOPHILA MELANOGASTER
}

\author{
M. E. YOUSIF and D. O. F. SKIBINSKI \\ Regional Ministry of Agriculture and Natural Resources, Juba, Sudan; Department of \\ Genetics, University College of Swansea, Singleton Park, Swansea SA2 8PP, U.K.
}

Received 22.iii.82

\section{SUMMARY}

\begin{abstract}
Artificial directional selection for increased sternopleural chaetae number in Drosophila melanogaster is compared with a mode of directional selection which incorporates an element of disruptive selection. Compared with directional selection, this directional-disruptive selection results in a similar response but with a lower selection differential, and higher realised heritability. The differences might be attributed to a higher genetic variance maintained by the directional-disruptive selection.
\end{abstract}

\section{INTRODUCTION}

ONE of the principal objectives in plant and animal breeding is to increase the rate of response of chosen characters under the influence of artificial selection. In populations of outbreeding organisms the response to selection can be predicted by the equation $R=i \sigma_{p} h^{2}$ or $R=S h^{2}$ where $R$ is response, $S$ is selection differential, $i$ is intensity of selection, $\sigma_{p}$ is phenotypic standard deviation and $h^{2}$ is heritability (Falconer, 1960). The final gain when the selection limit is reached is also dependent on effective population size (Robertson, 1960). The most effective forms of selection will in addition take into account information obtained from relatives' performance (Falconer, 1960).

In practise the response to selection may be improved to some extent by increasing $h^{2}$, by reducing the environmental variation, or by increasing $i$. The intensity of selection is however limited by the reproductive capacity of the organism and also by the need to avoid the genetic drift and inbreeding that may arise when small numbers of individuals are used as parents.

Attention has also been given to the possibility that population structure might influence the success of artificial selection. Wright has long argued that if a population is subdivided into a number of lines, selection within and between the lines coupled with occasional crossing among the lines will lead to more rapid improvement than selection in an undivided population (for example; Wright, 1978). A condition for the greater response in the subdivided population is that there be epistasis with respect to fitness. If variation at the loci for the character under selection is additive with respect to fitness the two modes of selection will not produce different responses. A number of experimental comparisons of selection in subdivided and undivided populations have been made: for example, Bowman and Falconer (1960) in mice, Enfield (1970) and Goodwill (1974) in Tribolium, and Madalena and Robertson (1975) and Rathie and Nicholas (1980) in Drosophila. In all cases subdivision did not result in any greater response to selection. 
This paper describes an attempt to increase the response to directional selection for increased sternopleural chaetae number in Drosophila melanogaster by artifically increasing the genetic variance and heritability through the use of disruptive selection. The definition of disruptive selection used here is that given by Thoday (1972) as selection for more than one value on a phenotypic scale within any one generation. Disruptive selection on continuous characters has been shown repeatedly in laboratory experiments to be capable of increasing genetic variance (for example; Gibson and Thoday, 1963; Scharloo, 1964). It is possible therefore that the response to directional selection might be enhanced by the incorporation of a certain amount of disruptive selection into the experimental design. This is done in the experiments described here by taking the most extreme individuals to provide one half of the selected parents, and taking individuals at random from the remaining scored progeny to make up the remainder of the selected parents. This is not analogous to the usual method of disruptive selection where the highest and lowest individuals are chosen as parents each generation.

The hypothesis to be tested is that while the selection differential may not be as high as it could be with conventional directional selection, the response may be greater because the disruptive element of the selection may maintain high genetic variation which can be exploited by the directional selection.

\section{MATERIALS AND EXPERIMENTAL DESIGN}

The selection lines were set up using a strain of Drosophila melanogaster called Chateau Tahbilk collected from a vineyard at Melborne, Australia in 1979. It has been maintained since then in a population cage at Swansea at $25^{\circ} \mathrm{C}$ and approximately 70 per cent relative humidity. The strain was chosen as it was known from previous experiments to have responded well to directional selection for sternopleural chaetae number.

Flies were raised in $25 \mathrm{~mm}$ diameter $\times 100 \mathrm{~mm}$ glass vials each containing $10 \mathrm{ml}$ of an agar food medium (Mittler and Bennet, 1962). Sternopleural chaetae number, was counted as the sum of the macro- and micro-chaetae on the left and right sides of the fly. Each selection line was set up with four vials each containing three unselected virgin females and three unselected males taken from the population cage. The progeny from these bottles are called the $G_{0}$ or base generation. The generation interval was 14 days and parents were left in vials for four days before being discarded. 20 male and 20 virgin female progeny were scored from each vial each generation (that is $4 \times 40=160$ progeny per selection line); parents were chosen from these progeny.

Three experimental designs were used:

\section{(i) Directional selection $(D)$}

Each generation, the twelve male and twelve female progeny having the highest chaetae numbers of the 80 of each sex scored, were selected as parents for the next generation. This design was replicated three times. 


\section{(ii) Directional-disruptive selection $(D D)$}

Each generation, the six male and six female progeny having the highest chaetae numbers out of the 80 progeny scored, were selected as parents, together with a further six male and six female progeny selected at random with respect to chaetae number from the 68 progeny remaining after the initial selection of the high chaetae number progeny. This design was replicated three times.

\section{(iii) Unselected control (C)}

Each generation twelve male and twelve female progeny were chosen at random as parents from progeny samples of 80 of each sex. Progeny from the control line were not scored every generation. This design was replicated twice.

A quasi-random mating system (Thoday and Boam, 1961; Gibson and Thoday, 1963) was used in which the 24 selected parents were distributed between four vials as shown below where $H$ stand for high and $R$ for random.

\begin{tabular}{|c|c|c|c|c|}
\hline $\begin{array}{c}\text { Experimental } \\
\text { Design }\end{array}$ & vial 1 & vial 2 & vial 3 & vial 4 \\
\hline Directional $(D)$ & $3 H \% ९+3 H \delta \delta^{\circ}$ & $3 H \% ९+3 H \delta \delta^{\circ}$ & $3 H \risingdotseq \supsetneq+3 H \delta^{\circ} \delta$ & $3 H \risingdotseq q+3 H \delta^{\circ} \delta$ \\
\hline $\begin{array}{l}\text { Directional- } \\
\text { Disruptive }(D D)\end{array}$ & $3 H \& \&+3 H$ ठేठ & $3 H \& q+3 R \delta^{\circ} \delta^{\circ}$ & $3 R \Re \&+3 H \delta^{\circ} \delta^{\circ}$ & $3 R q q+3 R \delta^{\circ} \delta^{\circ}$ \\
\hline Control $(C)$ & $3 R \& \&+3 R \delta^{8} \delta^{\prime}$ & $3 R \& \&+3 R \delta^{\prime} \delta^{\circ}$ & $3 R \Re \&+3 R \delta^{\circ} \delta^{\circ}$ & $3 R \wp q+3 R \delta^{*} \delta^{*}$ \\
\hline
\end{tabular}

Apart from the restrictions given above the selected parents were distributed between the vials at random. Throughout the experiment it was found that in the directional-disruptive lines, vials $2(H \times R)$ and $3(R \times$ $H)$, the "hybrid" cultures, together contributed about half of the high selected parents, the remainder coming almost exclusively from vial 1 $(H \times H)$. There was never danger of isolation of vials 1 and 4 as can happen when disruptive selection is for the highest and lowest flies each generation (Gibson and Thoday, 1964).

In the $D$ and $D D$ lines, selection was carried on for ten generations. From generations ten $\left(G_{10}\right)$ to thirteen $\left(G_{13}\right)$ the method of selection in the $D D$ lines was changed to that of the $D$ lines. That is from $G_{10}$ to $G_{13}$, both sets of lines were subjected to conventional directional selection for the highest flies each generation. The principal objective was to provide an estimate of the realised heritability in the $D$ and $D D$ lines at the end of the intitial ten generations of selection.

\section{Results}

The generation progeny means for the three experimental designs averaged over replicate lines are shown in fig. 1 . The coefficient of variation for the three experimental designs averaged over replicate lines are shown for each generation in fig. 2. Sexes have been combined. As the responses shown in fig. 1 appear to be approximately linear over the period of 


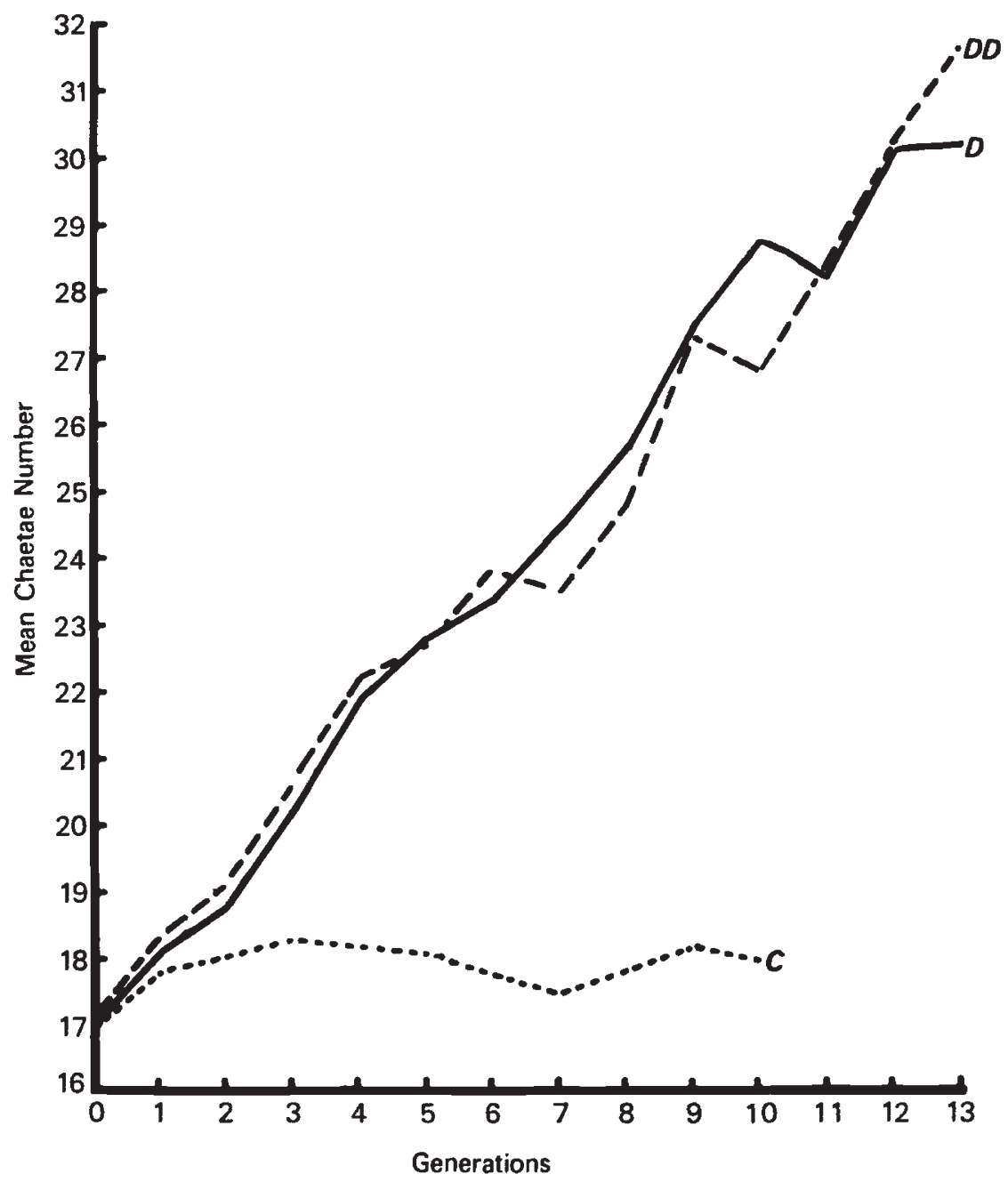

FIG. 1.-Progeny mean chaetae number averaged over replicates plotted against generation number for the directional $(D)$, directional-disruptive $(D D)$ and control $(C)$ lines.

selection, the average response to selection per generation $(R)$ for each selection line has been estimated as the slope of the regression of progeny mean chaetae number on generation number. The selection applied per generation is measured by the selection differential, which is the mean phenotypic value of the individuals selected as parents expressed as a deviation from the generation progeny mean. The average selection differential per generation $(S)$ is measured for each line as the regression of selection differential per generation number. Thus for the purposes of comparing the $D$ and $D D$ designs, $R$ and $S$ can be regarded as measures of the amount of response and amount of selection applied respectively. The realised heritability $\left(h^{2}\right)$ was also computed for each line as the regression of generation means on the accumulated selection differential. Values of $R, S$ and $h^{2}$ are shown in table 1 for each selection line for the 


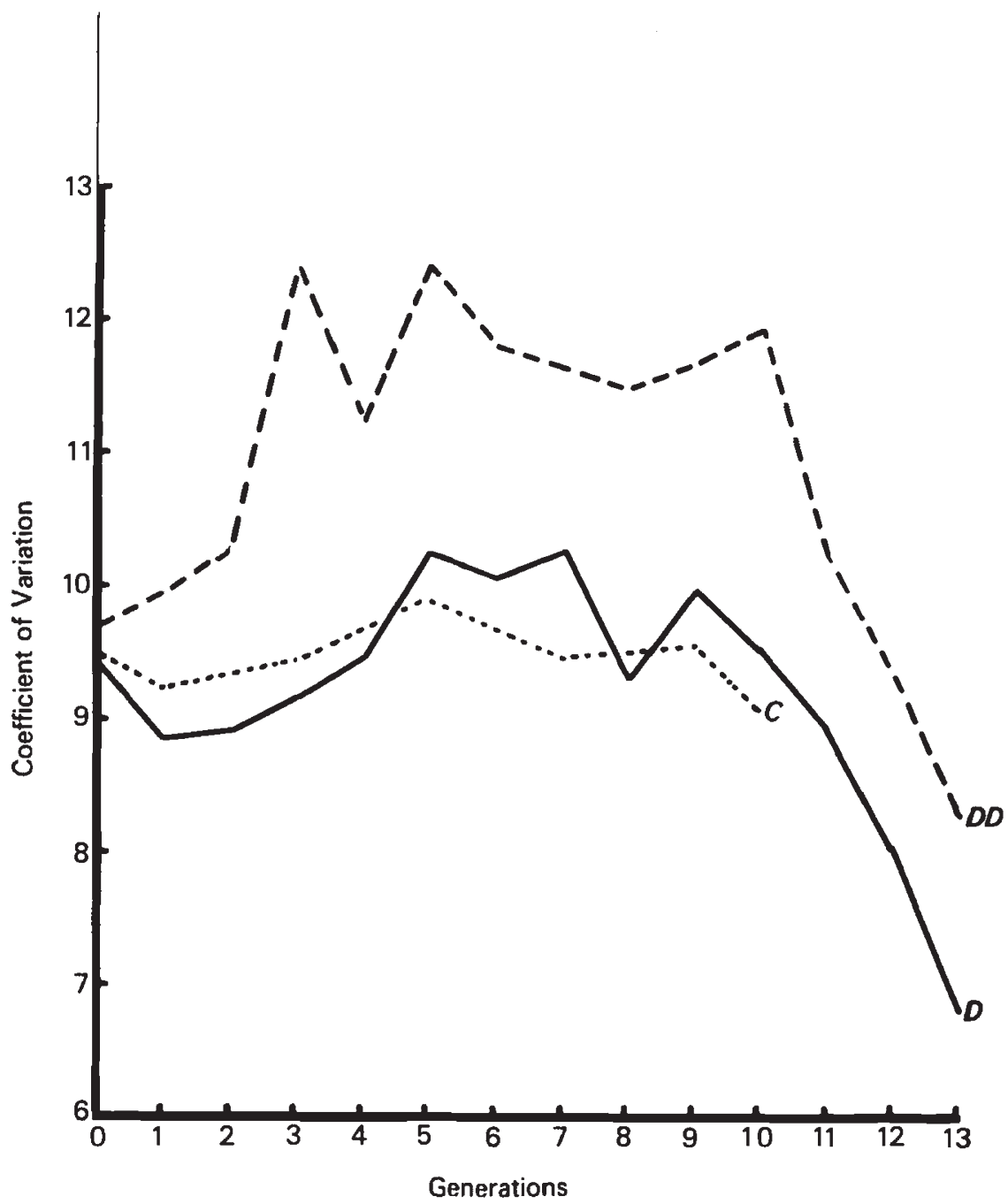

FIG. 2.-Coefficient of variation of progeny averaged over replicates plotted against generation number for the three experimental designs.

two periods of selection $G_{0}-G_{10}$ and $G_{10}-G_{13}$ and also for $G_{0}$ to $G_{13}$ inclusive. The mean values for the $D$ and $D D$ experimental designs have been compared with a one way analysis of variance where the main effect ( $D$ versus $D D$ ) has one, and the error four degrees of freedom. $F$ values and probability levels are shown in the table.

In generations $G_{0}$ to $G_{10}$ the average response is slightly higher in the $D$ than $D D$ lines although this difference is not significant. The average selection differential is however significantly greater in the $D$ lines. This suggests that the response to selection in the $D D$ lines was as great as in the $D$ lines even though the selection applied was significantly less. A similar response with a lower average selection differential should be reflected in a higher realised heritability. $h^{2}$ is indeed higher in the $D D$ 
than in the $D$ lines though the difference is not significant. Nevertheless there is good evidence that the difference in experimental design does result in a difference in the relationship between $R$ and $S$.

The lower selection differential in the $D D$ lines is the result of a proportion of parents being of relatively low chaetae number. The relatively high response despite this low selection differential could be explained if the amount of genetic variation available for selection was greater in the $D D$ than $D$ lines. This would also be consistent with the anticipated effect of directional-disruptive selection. Two sets of observations suggest that the genetic variation was indeed greater in the $D D$ lines. First, as seen in fig. 2 , the phenotypic variation was greater in the $D D$ lines in the period $G_{0}$ to $G_{10}$ than in the $D$ lines which maintained a similar level of variation to the controls. Although the values are not shown, the individual culture vials in the $D D$ lines showed the familiar pattern for disruptive selection in which the hybrid cultures (here $H \times R$ and $R \times H$ ) tend to have mean chaetae values lying between those of the non-hybrid cultures (here $\mathrm{H} \times H$ and $R \times R$ ). The variance of these culture means, which is certainly genetic, contributed a substantial amount of phenotypic variation which was absent in the $D$ lines. Second, in generations $G_{10}$ to $G_{13}$ the average response in the $D D$ lines was significantly greater than in the $D$ lines even though there was no significant difference in selection differential. Also the realised heritability was significantly greater in the $D D$ lines. These observations taken together with the evidence of higher phenotypic variation in the $D D$ lines suggest that the level of genetic variation at $G_{10}$ in these lines was significantly greater than in the $D$ lines. The rather dramatic reduction in phenotypic variation in the $D D$ lines in $G_{10}$ to $G_{13}$ could be the result of a reduction in genetic variation caused by the directional selection. A contemporary reduction occurred in the $D$ lines.

The conventional directional selection applied in $G_{10}$ to $G_{13}$ could be regarded as an integrai part of the experimental design in the $D D$ lines. That is, the two experimental designs could be regarded as directional selection (in the $D$ lines) and directional-disruptive selection followed by a few generations of directional selection (in the $D D$ lines). The analysis shown for $G_{0}$ to $G_{13}$ in table 1 shows that although the average selection differential in the $D D$ lines is significantly lower than in the $D$ lines, the average responses of the two experimental designs are almost identical. Just as in $G_{0}$ to $G_{10}$ this results in a higher realised heritability in the $D D$ lines although here the difference between $D$ and $D D$ is significant.

\section{Discussion}

The main conclusion to be drawn from the results is that although directional-disruptive selection causes no greater average response to selection than conventional directional selection, it achieves a similar response but with a lower selection differential. This difference results in a higher realised heritability in the directional-disruptive than directional lines. Unfortunately, this result does not immediately suggest any practical advantage to directional-disruptive selection. This is because the selection differential in the directional lines could only be "brought down" to the level obtained in the directional-disruptive lines by increasing the proportion of individuals selected as parents. This would immediately introduce 
TABLE 1

Average response (R), average selection differential (S), and realised heritability $\left(\mathrm{h}^{2}\right)$ for each of the Directional (D) and Directional-Disruptive (DD) selection lines. Mean values have been compared using a one way analysis of variance; $\mathrm{F}$ values and probability levels are given

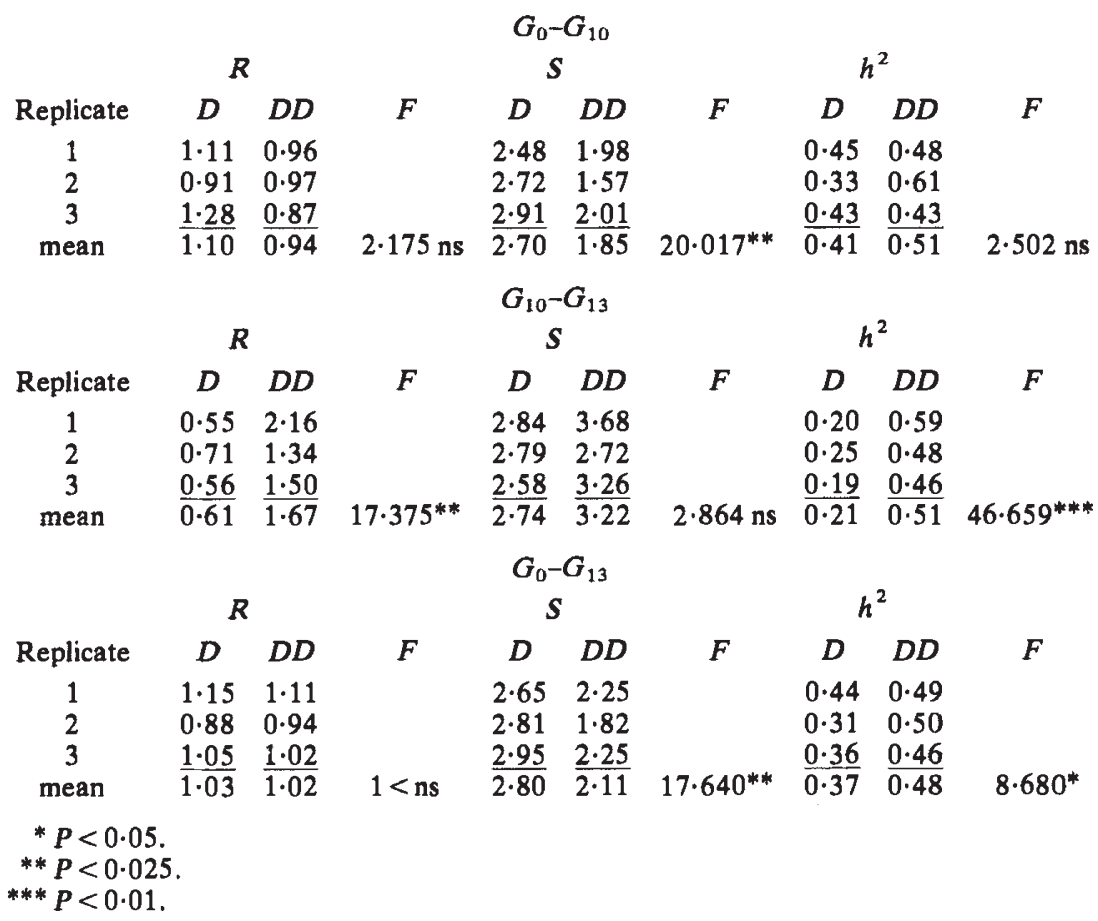

a confounding effect in comparing the effectiveness of the two experimental designs. Nevertheless there remains the possibility that some modifications to the experimental design might result in a greater overall response with directional-disruptive selection, given that the proportion of those progeny scored that are selected as parents, and the number of parents, are the same when comparing the two types of selection.

One possible advantage to directional-disruptive selection might relate to the influence of natural selection on the response to artificial selection. Continuous or quasi-continuous characters like sternopleural chaetae number are thought to be under stabilising selection, phenotypic extremes having lower fitness than phenotypic intermediates (Scossiroli, 1959; Barnes, 1968; Kearsey and Barnes, 1970). The intensity of conventional directional selection that can be applied in practice might therefore be limited to some extent by the lower fitness of phenotypically extreme phenotypes. Because the phenotypic variation in the directional-disruptive lines was higher and the selection differential lower than in the directional lines, the intensity of selection, measured as selection differential divided by phenotypic standard deviation, was considerably less in the former than the latter. If the selected parents are on average nearer to the mean with directional-disruptive selection the deleterious effect of natural selection might be less. In the present experiments numbers of progeny emerging 
from vials were recorded but no significant differences were observed between the two selection designs.

The observed differences in the relationship between response and selection differential between the directional and directional-disruptive lines accord with the observations of higher phenotypic and genetic variance in the latter than the former. It would be wrong, however, to jump too readily to the conclusion that the higher genetic and phenotypic variance promoted by the disruptive selection was the direct cause of the higher realised heritability. However, the hypothesis that there is a causal link can be made to seem more plausible by considering the possible effects of disruptive and directional selection on the genetic structure of the population. There is evidence that in populations not subject to artifical selection, increasing $(+)$ and decreasing $(-)$ alleles at different loci have a tendency to occur at high frequency in balanced repulsion combinations $(+-$ or -+$)$ (Gibson and Thoday, 1962; Thoday and Gibson, 1972). The predominance of such balanced combinations is in fact a predicted effect of stabilising selection (Wright, 1935). The response to directional selection will be expected to involve not only the increase in frequency of balanced or unbalanced combinations occurring in the base population but also the increase in frequency of unbalanced combinations $(++$ or --$)$ produced by recombination in repulsion heterozygotes (Thoday, Gibson, and Spickett, 1964). The response to disruptive selection has led to a variety of genetic polymorphisms of large effect including both balanced and unbalanced allele combinations (for example; Gibson and Thoday, 1962; Wolstenholme and Thoday, 1963). Thus, while directional selection will fix favourable balanced combinations, certain advantageous alleles may be selected against and lost if they are balanced by linked disadvantageous alleles of large effect. If an element of disruptive selection is included, such advantageous alleles may be maintained for long enough in the population for unfavourable linkages to be broken. In this light, directional-disruptive selection may not only give a greater short term response than conventional directional selection but also a greater final gain at the selection limit.

Acknowledgments.-Grateful thanks are due to the Regional Government of the Southern Sudan who sponsored M. E. Yousif during this study. We would also like to thank Mrs M. Warren for technical assistance.

\section{REFERENCES}

BARNES, B. W. 1968. Stabilising selection in D. melanogaster. Heredity, 23, 433-443.

BOWMAN, J. C., AND FALCONER, D. S. 1960. Inbreeding depression and heterosis of litter size in mice. Genetical Research, 1, 262-274.

ENFIELD, F. D. 1970. Effect of population structure on progress from selection in Tribolium. Journal of Animal Science, 31, 163.

FALCONER, D.S. 1960. An Introduction to Quantitative Genetics. Oliver and Boyd, Edinburgh. GIBSON, J. B., AND THODAY, J. M. 1962. Effects of disruptive selection. VI. A second chromosome polymorphism. Heredity, 17, 1-26.

GIBSON, J. B., AND THODAY, J. M. 1963. Imposed quasi-random mating. Heredity, 18, 513-524.

GIBSON, J. B., AND THODAY, J. M. 1964. Effects of disruptive selection. IX. Low selection intensity. Heredity, 19, 125-130.

GOODWILL, R. 1974. Comparison of three selection programs using Tribolium castaneum. Journal of Heredity, 65, 8-14. 
KEARSEY, M. J., AND BARNES, B. W. 1970. Variation for metrical characters in Drosophila populations. II. Natural Selection. Heredity, 25, 11-21.

MADALENA, F, E., AND ROBERTSON, A. 1975. Population structure in artifical selection: studies with Drosophila melanogaster. Genet. Res., 24, 113-126.

MITTLER, S., AND BENNET, J. 1962. A simple food medium that requires no live yeast with the minimum of variables. Dros. Inf. Serv., 36, 131-132.

RATHIE, K. A., AND NICHOLAS, F. W. 1980. Artificial selection with differing population structures. Genet. Res., 36, 117-131.

ROBERTSON, A. 1960. A theory of limits in artificial selection. Proc. Roy. Soc. B, 153, 234-49.

SCHARLOO, W. 1964. The effect of disruptive and stabilising selection on the expression of a cubitus interruptus mutant in Drosophila. Genetics, 50, 553-562.

SCOSSIROLI, R. E. 1959. Selezione artificiale per un carrattere quantitative in popolazione di Drosophila melanogaster irradiate con raggi. X. Commitoto Nazionale per Recher. Nucleari, Divisione Biologica (CNB-4). Roma.

ThODAY, J. M. 1972. Disruptive selection. Proc. Roy. Soc. B, 182, 109-143.

THODAY, J. M., AND BOAM, T. B. 1961. Effects of disruptive selection, V. Quasi random mating. Heredity, 16, 219-223.

THODAY, J. M., AND GIBSON, J. B. 1972 A simple test for stabilising and disruptive selection. Egyptian Joumal of Genetics and Cytology, 1, 47-50.

THODAY, J. M., GIBSON, J. B., AND SPICKETT, S. G. 1964. Regular responses to selection. 2. Recombination and accelerated response. Genet. Res., 5, 1-19.

WOLSTENHOLME, D. R., AND THODAY, J, M. 1963. Effects of disruptive selection. VII. A third chromosome polymorphism. Heredity, 18, 413-431.

WRIGHT, s. 1935. The analysis of variance and the correlations between relatives with respect to deviations from an optimum. J. Genet., 30, 243-256.

WRIGHT, s. 1978. The relation of livestock breeding to theories of evolution. Journal of Animal Science, 46, 1192-1200. 